\title{
On Scalable Algorithms for Numerical Solution of Variational Inequalities Based on FETI and Semi-monotonic Augmented Lagrangians
}

\author{
Zdeněk Dostál ${ }^{1}$ and David Horák ${ }^{1}$ \\ VŠB-Technical University Ostrava, Applied Mathematics Tr17.listopadu, \\ CZ-70833, Ostrava, CzechRepublic, zdenek.dostal@vsb.cz, david.horak@vsb.cz
}

Summary. Theoretical and experimental results concerning a new FETI based algorithm for numerical solution of variational inequalities are reviewed. A discretized model problem is first reduced by the duality theory of convex optimization to the quadratic programming problem with bound and equality constraints. The latter is then optionally modified by means of orthogonal projectors to the natural coarse space introduced by Farhat and Roux in the framework of their FETI method. The resulting problem is then solved by a new variant of the augmented Lagrangian type algorithm with the inner loop for the solution of bound constrained quadratic programming problems. Recent theoretical results are reported that guarantee scalability of the algorithm. The results are confirmed by numerical experiments.

\section{Introduction}

The FETI method was originally proposed by Farhat and Roux [1992] for parallel solution of linear problems described by elliptic partial differential equations. Its key ingredient is decomposition of the spatial domain into nonoverlapping subdomains that are "glued" by Lagrange multipliers, so that after eliminating the primal variables, the original problem is reduced to a small, relatively well conditioned, typically equality constrained quadratic programming problem that is solved iteratively. Observing that the equality constraints may be used to define so called "natural coarse grid", Farhat et al. [1994] modified the basic FETI algorithm so that they were able to prove its numerical scalability.

If the FETI procedure is applied to an elliptic variational inequality, the resulting quadratic programming problem has not only the equality constraints, but also the non-negativity constraints. Even though the latter is a considerable complication as compared with the linear problem, the resulting problem is still easier to solve than the contact problem in displacements as it is smaller, better conditioned and only bound constrained. Promising experimental results by Farhat and Dureisseix [2002], who used a coarse grid initial 
approximation, supported this claim and even indicated numerical scalability of their method. Scalability was later proved for an algorithm that combined FETI with optimal dual penalty Dostál and Horák [2003b]. A different approach yielding experimental evidence of scalability was based on the augmented Lagrangian method was used by Dostál et al. [2000a,b], Dostál and Horák [2003a]. It should be noted that the effort to develop scalable solvers for variational inequalities was not restricted to FETI. For example, using ideas related to Mandel [1984], Kornhuber [1997], Kornhuber and Krause [2001], Wohlmuth and Krause. [2002] gave an experimental evidence of numerical scalability of the algorithm based on monotone multigrid. Probably the first theoretical results concerning development of scalable algorithms were proved by Schoeberl [1998b,a].

Here we review recent improvements to show scalability for this type of algorithms. We start our exposition by describing a simple model problem and the FETI methodology Dostál et al. [2000a] that turns the variational inequality into the quadratic programming problem with bound and equality constraints. Then we briefly review recent results concerning a new variant of the augmented Lagrangian method. Finally we report the results of numerical experiments that are in agreement with the theory and indicate high and numerical scalability of the algorithm presented.

\section{Model problem}

Let $\Omega=\Omega^{1} \cup \Omega^{2}, \Omega^{1}=(0,1) \times(0,1)$ and $\Omega^{2}=(1,2) \times(0,1)$ denote open domains with boundaries $\Gamma^{1}, \Gamma^{2}$ and their parts $\Gamma_{u}^{i}, \Gamma_{f}^{i}, \Gamma_{c}^{i}$ formed by the sides of $\Omega^{i}, i=1,2$, so that $\Gamma_{u}^{1}=\{0\} \times(0,1), \Gamma_{u}^{2}=\{2\} \times(0,1), \Gamma_{c}^{i}\{1\} \times(0,1)$, and $\Gamma_{f}^{i}$ are formed by the other sides of $\Omega^{i}, i=1,2$. Let $H^{1}\left(\Omega^{i}\right), i=1,2$ denote the Sobolev space of the first order in the space $L^{2}\left(\Omega^{i}\right)$ of functions on $\Omega^{i}$ whose squares are integrable in the sense of Lebesgue. Let

$$
V^{i}=\left\{v^{i} \in H^{1}\left(\Omega^{i}\right): v^{i}=0 \quad \text { on } \quad \Gamma_{u}^{i}\right\}
$$

denote the closed subspaces of $H^{1}\left(\Omega^{i}\right), i=1,2$, and let

$$
V=V^{1} \times V^{2} \quad \text { and } \quad \mathcal{K}=\left\{\left(v^{1}, v^{2}\right) \in V: v^{2}-v^{1} \geq 0 \quad \text { on } \quad \Gamma_{c}\right\}
$$

denote the closed subspace and the closed convex subset of $\mathcal{H}=H^{1}\left(\Omega^{1}\right) \times$ $H^{1}\left(\Omega^{2}\right)$, respectively. The relations on the boundaries are in terms of traces. On $\mathcal{H}$ we shall define a symmetric bilinear form

$$
a(u, v)=\sum_{i=1}^{2} \int_{\Omega^{i}}\left(\frac{\partial u^{i}}{\partial x} \frac{\partial v^{i}}{\partial x}+\frac{\partial u^{i}}{\partial y} \frac{\partial v^{i}}{\partial y}\right) d \Omega
$$

and a linear form 


$$
\ell(v)=\sum_{i=1}^{2} \int_{\Omega^{i}} f^{i} v^{i} d \Omega,
$$

where $f^{i} \in L^{2}\left(\Omega^{i}\right), i=1,2$ are the restrictions of

$f(x, y)=\left\{\begin{array}{cc}-3 \text { for } \quad(x, y) \in(0,1) \times[0.75,1) \\ 0 \quad \text { for } \quad(x, y) \in(0,1) \times[0,0.75) \text { and }(x, y) \in(1,2) \times[0.25,1) \\ -1 \text { for }(x, y) \in(1,2) \times[0,0.25)\end{array}\right\}$.

Thus we can define a problem to find

$$
\min \quad q(u)=\frac{1}{2} a(u, u)-\ell(u) \text { subject to } u \in \mathcal{K} .
$$

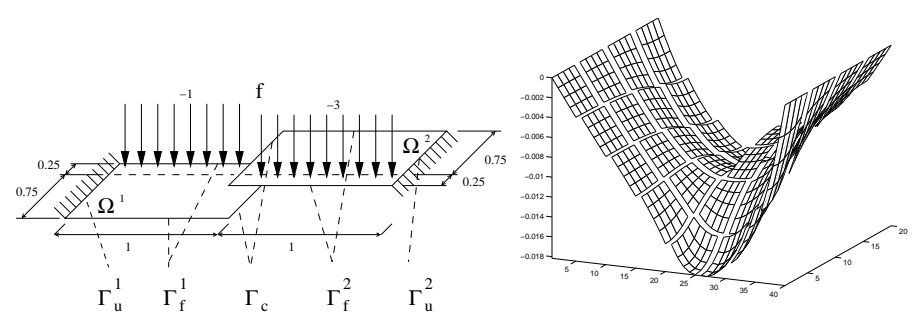

Fig. 1. Model problem and its solution

The solution of the model problem may be interpreted as the displacement of two membranes under the traction $f$. The membranes are fixed on the outer edges as in Figure 1 and the left edge of the right membrane is not allowed to penetrate below the right edge of the left membrane. Since the Dirichlet conditions are prescribed on parts $\Gamma_{u}^{i}, i=1,2$ of the boundaries with positive measure, the quadratic form $a$ is coercive which guarantees existence and uniqueness of the solution Hlaváček et al. [1988].

\section{Domain decomposition and discretized problem with a natural coarse grid}

To enable efficient application of the domain decomposition methods, we can optionally decompose each $\Omega^{i}$ into square subdomains $\Omega^{i 1}, \ldots, \Omega^{i p}, p=s^{2}>$ $1, i=1,2$. The continuity in $\Omega^{1}$ and $\Omega^{2}$ of the global solution assembled from the local solutions $u^{i j}$ will be enforced by the "gluing" conditions $u^{i j}(x)=$ $u^{i k}(x)$ that should be satisfied for any $x$ in the interface $\Gamma^{i j, i k}$ of $\Omega^{i j}$ and $\Omega^{i k}$. After modifying appropriately the definition of problem (1), introducing regular grids in the subdomains $\Omega^{i j}$ that match across the interfaces $\Gamma^{i j, k l}$, indexing contiguously the nodes and entries of corresponding vectors in the 
subdomains, and using the finite element discretization, we get the discretized version of problem (1) with the auxiliary domain decomposition that reads

$$
\min \frac{1}{2} u^{\top} A u-f^{\top} u \quad \text { s.t. } \quad B^{I} u \leq 0 \quad \text { and } \quad B^{E} u=0 .
$$

In (2), $A$ denotes a positive semidefinite stiffness matrix, the full rank matrices $B^{I}$ and $B^{E}$ describe the discretized inequality and gluing conditions, respectively, and $f$ represents the discrete analog of the linear term $\ell(u)$. Denoting

$$
\lambda=\left[\begin{array}{c}
\lambda^{I} \\
\lambda^{E}
\end{array}\right] \quad \text { and } \quad B=\left[\begin{array}{c}
B^{I} \\
B^{E}
\end{array}\right],
$$

we can write the Lagrangian associated with problem (2) briefly as

$$
L(u, \lambda)=\frac{1}{2} u^{\top} A u-f^{\top} u+\lambda^{\top} B u .
$$

It is well known that (2) is equivalent to the saddle point problem

$$
\text { Find }(\bar{u}, \bar{\lambda}) \quad \text { s.t. } L(\bar{u}, \bar{\lambda})=\sup _{\lambda_{I} \geq 0} \inf _{u} L(u, \lambda) .
$$

After eliminating the primal variables $u$ from (3), we shall get the minimization problem

$$
\min \Theta(\lambda) \quad \text { s.t. } \quad \lambda_{I} \geq 0 \quad \text { and } \quad R^{\top}\left(f-B^{\top} \lambda\right)=0,
$$

where

$$
\Theta(\lambda)=\frac{1}{2} \lambda^{\top} B A^{\dagger} B^{\top} \lambda-\lambda^{\top} B A^{\dagger} f
$$

$A^{\dagger}$ denotes a generalized inverse that satisfies $A A^{\dagger} A=A$, and $R$ denotes the full rank matrix whose columns span the kernel of $A$. We shall choose $R$ so that its entries belong to $\{0,1\}$ and each column corresponds to some floating auxiliary subdomain $\Omega^{i j}$ with the nonzero entries in the positions corresponding to the indices of nodes belonging to $\Omega^{i j}$.

Even though problem (4) is much more suitable for computations than (2), further improvement may be achieved by adapting some simple observations and the results of Farhat et al. [1994], Mandel and Tezaur [1996]. Let us denote

$$
F=B A^{\dagger} B^{\top}, \quad \widetilde{G}=R^{\top} B^{\top}, \quad \widetilde{e}=R^{\top} f, \quad \widetilde{d}=B A^{\dagger} f,
$$

and let $\widetilde{\lambda}$ solve $\widetilde{G} \widetilde{\lambda}=\widetilde{e}$, so that we can transform the problem (4) to minimization on the subset of the vector space by looking for the solution in the form $\lambda=\mu+\widetilde{\lambda}$. Since

$$
\frac{1}{2} \lambda^{\top} F \lambda-\lambda^{\top} \widetilde{d}=\frac{1}{2} \mu^{\top} F \mu-\mu^{\top}(\widetilde{d}-F \widetilde{\lambda})+\frac{1}{2} \tilde{\lambda}^{\top} F \widetilde{\lambda}-\widetilde{\lambda}^{\top} \tilde{d}
$$

problem (4) is, after returning to the old notation, equivalent to 


$$
\min \quad \frac{1}{2} \lambda^{\top} F \lambda-\lambda^{\top} d \quad \text { s.t } \quad G \lambda=0 \quad \text { and } \quad \lambda^{I} \geq-\widetilde{\lambda}^{I}
$$

where $d=\widetilde{d}-F \widetilde{\lambda}$ and $G=T \widetilde{G}$ denotes a matrix arising from the orthonormalization of the rows of $\widetilde{G}$ by the Schmidt process defined by the regular matrix $T$.

Our final step is based on observation that the problem (6) is equivalent to

$$
\min \quad \frac{1}{2} \lambda^{\top} P F P \lambda-\lambda^{\top} P d \quad \text { s.t } \quad G \lambda=0 \quad \text { and } \quad \lambda^{I} \geq-\widetilde{\lambda}^{I}
$$

where

$$
Q=G^{\top} G \quad \text { and } \quad P=I-Q
$$

denote the orthogonal projectors on the image space of $G^{\top}$ and on the kernel of $G$, respectively, so that $P \lambda=\lambda$ for feasible $\lambda$.

\section{Semi-monotonic augmented Lagrangian method and scalability}

In this section we shall describe a recent modification Dostál [2003] of the algorithm introduced earlier by Dostál et al. [2003]. The algorithm treats each type of constraints separately, so that efficient algorithms using projections and adaptive precision control Dostál and Schoeberl [2004] may be used for the bound constrained QP problems.

Let us recall that the augmented Lagrangian for (7) and its gradient are given by

$$
\begin{gathered}
L(\lambda, \mu, \rho)=\frac{1}{2} \lambda^{\top} P F P \lambda-\lambda^{\top} P d+\mu^{\top} G \lambda+\frac{1}{2} \rho\|Q \lambda\|^{2} \\
g(\lambda, \mu, \rho)=P F P \lambda-P d+G^{\top}(\mu+\rho G \lambda) .
\end{gathered}
$$

The projected gradient $g^{P}=g^{P}(\lambda, \mu, \rho)$ of $L$ at $\lambda$ is given componentwise by

$$
g_{i}^{P}=g_{i} \text { for } \lambda_{i}>-\bar{\lambda}_{i} \text { or } i \notin \mathcal{I} \text { and } g_{i}^{P}=g_{i}^{-} \text {for } \lambda_{i}=-\bar{\lambda}_{i} \text { and } i \in \mathcal{I}
$$

with $g_{i}^{-}=\min \left(g_{i}, 0\right)$, where $\mathcal{I}$ is the set of indices of constrained entries of $\lambda$. The Hessian of the augmented Lagrangian $L(\lambda, \mu, \rho)$ is given by $H^{\rho}=P F P+\rho Q$.

Algorithm 1. Semi-monotonic augmented Lagrangian method (SALM).

$$
\begin{array}{ll}
\text { Step 0. } & \text { Set } \eta>0,1<\beta, \rho_{0}>0, M>0, \mu^{0} \text { and } k=0 . \\
\text { Step 1. } & \text { Find } \lambda^{k} \text { so that }\left\|g^{P}\left(\lambda^{k}, \mu^{k}, \rho_{k}\right)\right\| \leq \min \left\{M\left\|G \lambda^{k}\right\|, \eta\right\} . \\
\text { Step 2. } & \text { If }\left\|g^{P}\left(\lambda^{k}, \mu^{k}, \rho_{k}\right)\right\| \text { and }\left\|G \lambda^{k}\right\| \text { are sufficiently small, then stop. } \\
\text { Step 3. } & \mu^{k+1}=\mu^{k}+\rho_{k} G \lambda^{k} \\
\text { Step 4. } & \text { If } L\left(\lambda^{k+1}, \mu^{k+1}, \rho_{k+1}\right)<L\left(\lambda^{k}, \mu^{k}, \rho_{k}\right)+\frac{\rho_{k+1}}{2}\left\|G \lambda^{k+1}\right\|^{2}
\end{array}
$$


Step $4 a$. then $\rho_{k+1}=\beta \rho_{k}$

Step $4 b$. else $\rho_{k+1}=\rho_{k}$

end if.

Step 5. Increase $k$ by one and return to Step 1.

An implementation of Step 1 is carried out by the minimization of the augmented Lagrangian $L$ subject to $\lambda_{I} \geq-\bar{\lambda}_{I}$ by the MPRGP algorithm Dostál and Schoeberl [2004]. The MPRGP algorithm with the choice of parameters $\Gamma=1$ and $\bar{\alpha} \in\left(0,\left\|H^{\rho}\right\|^{-1}\right]$ generates the iterations $\left\{\widetilde{\lambda}^{k i}, i=1,2, \ldots\right\}$ for the unique solution $\bar{\lambda}^{k}$ of the auxiliary minimization problem so that the rate of convergence in the energy norm defined by $\|\lambda\|_{H^{\rho}}^{2}=\lambda^{\top} H^{\rho} \lambda$ may be expressed by means of the least eigenvalue $\alpha_{1}$ of $\left\|H^{\rho}\right\|$ in the form

$$
\left\|\widetilde{\lambda}^{k i}-\bar{\lambda}^{k}\right\|_{H^{\rho}}^{2} \leq \frac{2 \eta^{i}}{\alpha_{1}}\left(L\left(\widetilde{\lambda}^{k 0}, \mu^{k}, \rho_{k}\right)-L\left(\bar{\lambda}^{k}, \mu^{k}, \rho_{k}\right)\right), \quad \eta=1-\frac{\bar{\alpha} \alpha_{1}}{4} .
$$

Algorithm 1 has been proved Dostál [2003] to converge for any set of parameters that satisfy the prescribed relations. It has also been shown that if $\rho_{k} \geq M^{2} / \alpha_{1}$, then

$$
L\left(\lambda^{k+1}, \mu^{k+1}, \rho_{k+1}\right) \geq L\left(\lambda^{k}, \mu^{k}, \rho_{k}\right)+\frac{\rho^{k+1}}{2}\left\|G \lambda^{k+1}\right\|^{2},
$$

so that it is possible to give an upper bound on $\rho^{k}$ in terms of $\alpha_{1}$. The experiments have shown that the penalty parameter should be sufficiently high to enforce fast convergence of the outer loop. Let us recall that a large penalty parameter need not delay too much the convergence of the inner loop as the image spaces of the projectors $P$ and $Q$ are invariants subspaces of $H^{\rho}$ so that the arguments of Dostál [1999] may be applied. Moreover, it has been proved Dostál [2003] that there is a bound on the number of outer iterations that are necessary to achieve prescribed relative feasibility error $\epsilon\|P d\|$. The bound may be expressed in terms of the extreme eigenvalues of the Hessian of the augmented Lagrangian. Since it has been established by Mandel and Tezaur [1996] and more recently by Klawonn and Widlund [2001] that the smallest eigenvalue and the spectral condition number of the restriction of $P F P$ to the kernel of $G$ are $O(1)$ for fixed ratio $H / h$, it is possible to prove the following theorem.

Theorem 1. Let $C, \rho$ and $\epsilon$ denote given positive numbers, and let $\left\{\lambda_{H, h}^{k}\right\},\left\{\mu^{k}\right\}$ and $\left\{\rho_{k}\right\}$ be generated by Algorithm 1 with Step 1 implemented by the MPRGP algorithm initiated by $\lambda_{H, h}^{0}=0, \mu^{0}=0, \eta=\|P d\|$ and $\rho_{0}>0$ for the solution of the problem (7) arising from the regular discretization of (1) with the decomposition and discretization parameters $H$ and $h$, respectively. Then there is an integer $k$ independent of $h$ and $H$ such that $H / h \leq C$ implies

$$
\left\|g^{P}\left(\lambda_{H, h}^{k}, \mu^{k}, \rho_{k}\right)\right\| \leq \epsilon\|P d\| \quad \text { and } \quad\left\|G \lambda_{H, h}^{k}\right\| \leq \epsilon\|P d\| .
$$


We have implemented Algorithm 1 for solution of (1). Results of computations to the relative precision 1e-4 are in Table 1 . The largest problem discretized by more than two million nodal variables required 167 seconds of 32 processors of SGI Origin. The finest discretization of (1) that we have run so far comprised 8464272 nodal variables and its solution required 65 iterations and 1281 seconds of 64 processors with decomposition into 128 subdomains. The results are the same as in Dostál and Horák [2003a,b] as no update of the penalty parameter was observed in either case.

Table 1. Numerical scalability of $\mathrm{AL}$ for $\mathrm{H} / \mathrm{h}=128$ and $\rho=1 \mathrm{e}+3$

\begin{tabular}{|l|c|c|c|c|}
\hline dimension & 33282 & 133128 & 532512 & 2130048 \\
\hline subdomains & 2 & 8 & 32 & 128 \\
\hline iterations & 28 & 59 & 36 & 47 \\
\hline
\end{tabular}

\section{Comments and conclusion}

We have reviewed our recent results related to application of the augmented Lagrangians with the FETI based domain decomposition method to the solution of variational inequalities using recently developed algorithms for the solution of special QP problems. In particular, we have shown that the solution of the discretized problem to a prescribed precision may be found in a number of iterations bounded independently of the discretization parameter. Numerical experiments with the model variational inequality are in agreement with the theory and indicate that the algorithm may be efficient. Let us point out that similar development may be done also on the ground of DP-FETI. We shall describe it elsewhere together with applications to contact problems of elasticity.

Acknowledgement. This research is supported by grants No. 101/02/0072 and 101/01/0538 of the Grant Agency of the Czech Republic and by projects of the Ministry of Education No. AVOZ2076919 and ME641.

\section{References}

Z. Dostál. On preconditioning and penalized matrices. Num. Lin. Alg. Appl., 6: 109-114, 1999.

Z. Dostál. Inexact semi-monotonic augmented Lagrangians with optimal feasibility convergence for quadratic programming with simple bounds and equality constraints. submitted to SIAM J. Num. Anal., 2003. 
Z. Dostál, A. Friedlander, and S. Santos. Augmented Lagrangians with adaptive precision control for quadratic programming with simple bounds and equality constraints. SIAM J. Opt., 13:1120-1140, 2003.

Z. Dostál, F. Gomes, and S. Santos. Duality based domain decomp. with natural coarse space for variat. ineq. J. Comput. Appl. Math., 126:397-415, 2000a.

Z. Dostál, F. Gomes, and S. Santos. Solution of contact problems by FETI domain decomp. Comput. Meth. Appl. Mech. Eng., 190:1611-1627, 2000b.

Z. Dostál and D. Horák. Scalability and FETI based algorithm for large discretized variational inequalities. Math. and Comput. in Simul., 61:347-357, 2003a.

Z. Dostál and D. Horák. Scalable FETI with optimal dual penalty for a variational inequality. to appear in Num. Lin. Alg. and Appl., 2003b.

Z. Dostál and J. Schoeberl. Minimizing quadratic functions subject to bound constraints with the rate of convergence and finite termination. to appear in Comput. Optimiz. and Appl., 2004.

C. Farhat and D. Dureisseix. A numerically scalable domain dec. meth. for solution of frictionless contact problems. to appear in Int. J. Num. Meth. Eng., 2002.

C. Farhat, J. Mandel, and F. Roux. Optimal convergence properties of the FETI domain decomp. method. Comp. Meth. Appl. Mech. Eng., 115:367-388, 1994.

C. Farhat and F. Roux. An unconventional domain decomposition method for an efficient parallel solution of large-scale finite element systems. SIAM J. Sc. Stat. Comput., 13:379-396, 1992.

I. Hlaváček, J. Haslinger, J. Nečas, and J. Lovíšek. Solution of Variational Inequalities in Mechanics. Springer Verlag Berlin, 1988.

A. Klawonn and O. Widlund. FETI and Neumann-Neumann iterative substructuring methods: connections and new results. Communic. on Pure and Appl. Math., LIV:57-90, 2001.

R. Kornhuber. Adaptive Monotone Multigrid Methods for Nonlinear Variational Problems. Teubner, Stuttgart, 1997.

R. Kornhuber and R. Krause. Adaptive multigrid methods for Signorini's problem in linear elasticity. Comput. Visualiz. in Science, 4:9-20, 2001.

J. Mandel. Étude algébrique d'une méthode multigrille pour quelques problèmes de frontière libre. Compt. Rendus de l'Acad. des Scien., pages 469-472, 1984.

J. Mandel and R. Tezaur. Convergence of a Substructuring Method with Lagrange Multipliers. Numer. Math., 73:473-487, 1996.

J. Schoeberl. Efficient contact solvers based on domain decomposition techniques. Comput. and Math., 42:1217-1228, 1998a.

J. Schoeberl. Solving the Signorini problem on the basis of domain decomposition techniques. Part. Diff. Eqs. in Physics and Biology, 60:323-344, 1998b.

B. Wohlmuth and R. Krause. Monotone methods on nonmatching grids for nonlinear contact problems. Technical report, 2002. Research Report No. 2002/02 of the Stuttgart University, Sonderforsungsbereich 404. 